\title{
Three-dimensional boundary element method model in the frequency domain for simulating dynamic heat conduction
}

\author{
J. Prata ${ }^{1}$, A. Tadeu ${ }^{2}$ \& N. Simões ${ }^{2}$ \\ ${ }^{I}$ ITeCons - Institute for Research and Technological Development in \\ Construction Sciences, Portugal \\ ${ }^{2}$ Department of Civil Engineering, University of Coimbra, Portugal
}

\begin{abstract}
The boundary element method (BEM) is a suitable numerical method used to study heat diffusion by conduction, which only requires the discretization of the boundary of the material discontinuities. However, one of the biggest challenges to this technique is the integration of mathematical singularities. In this work the $\mathrm{BEM}$ is used to simulate the heat conduction in the vicinity of $3 \mathrm{D}$ inclusions, when heated by dynamic heat point sources. Continuity of temperatures and heat fluxes are prescribed along the boundary interfaces. All boundary interfaces are discretized using constant elements. The proposed algorithm is verified by means of known analytical solutions for cylindrical inclusions.

Keywords: heat conduction, three-dimensional sources, three-dimensional boundary element method, singular integrals.
\end{abstract}

\section{Introduction}

The study of heat transfer has been increasingly important in many branches of engineering. Numerous works have been published proposing different models, based on both analytical [1] and numerical analysis, to study heat diffusion through different systems and materials. Different approaches could be taken into account in a numerical analysis, depending whether the numerical method is based on domain-discretization such as finite elements (FEM) [2] and finite differences (FDM) [3, 4] or on boundary discretization such as the boundary element method (BEM) [5, 6]. More recently, some researchers have been focused on the development of meshless methods to study physical phenomenon 
of heat transfer, requiring neither domain nor boundary discretization, such as the method of fundamental solutions (MFS) [7].

Amongst the numerical methods, the BEM is possibly one of the most suitable tools for modeling homogeneous unbounded or semi-infinite systems because it automatically satisfies the far field conditions. As a result the BEM enables a compact description of the regions, discretizing only the boundaries of the inclusions, resulting however in fully populated systems of equations, contrary to the sparse systems given by the FDM and FEM techniques. Thus, the size of the equation system to be solved is efficiently reduced. One disadvantage of the BEM is that it can only be applied to more general geometries and media when the relevant fundamental solution is known. However, this may not always be possible. Furthermore it is well known that, depending on the distance between the source point and the node being integrated, the boundary integrals may become singular or nearly singular, increasing the difficulty associated with the mathematical model.

Due to the high computational cost brought by three-dimensional simulation phenomena, different authors have proposed a number of BEM formulations. Ma et al. [8] applied a BEM formulation to study transient heat conduction in 3D solids with fiber inclusions. Jablonski [9] solved 3D Laplace and Poisson equations by proposing the analytical evaluation of the surface integrals appearing in BEMs. Qin et al. [10] implemented changes to the conventional distance transformation technique to evaluate nearly singular integrands on 3D boundary elements, including planar and curved surface elements and very irregular elements of slender shape.

One of the biggest challenges of the BEM is the correct integration of the singular integrals. The BEM research community has been proposing a variety of methods in order to overcome some of the difficulties posed by singularities, as it is described by Zhou et al. [11]. Alternatively it can be used analytical and semi-analytical methods, as well as other approaches such as non-linear transformation [12-14] or distance transformation techniques [15-17].

The accuracy of the BEM is highly dependent on the precision of singular integrals which, for 3D problems, are mostly solved using numerical schemes based on Gaussian integration schemes. However, some researchers have been looking for semi-analytical solutions or sophisticated approaches in order to improve the accuracy of BEM models used to solve specific problems [18]. Niu et al. [19] proposes a semi-analytical algorithm for 3D elastic problems that require the evaluation of nearly strongly singular and hypersingular integrals on the triangular and quadrilateral elements. Applying a scheme of integration by parts, the nearly singular surface integrals are transformed to a set of line integrals along the boundary for which standard numerical quadrature can be used.

This paper first presents a 3D BEM formulation in the frequency domain to simulate the heat diffusion inside a 3D inclusion containing an inner 3D body/defect. The analytical evaluation of the singular integral that appear in the 3D BEM formulation, when the element being integrated is the loaded one, is given. These functions are subsequently incorporated in the 3D BEM 
formulation and used to simulate the heat diffusion in the vicinity of a 3D circular cylindrical inclusion containing an embedded circular cylindrical body, when heated by a dynamic point source. Analytical solutions have been derived for this problem.

\section{Geometry of the problem}

Consider a 3D body (Medium 1) bounded by a surface $S_{1}$, implanted in a 3D inclusion (Medium 2) with surface $\mathrm{S}_{2}$, which is surrounded by a spatially uniform solid medium (Medium 3) with thermal diffusivity $K_{3}$ (see Figure 1). Media 1 and 2 exhibit thermal diffusivities $K_{1}$ and $K_{2}$, respectively. Thermal diffusivity $K_{n}$ is defined by $\frac{\lambda_{n}}{\rho_{n} c_{n}}$, where $\lambda_{n}$ is the thermal conductivity, $\rho_{n}$ is the density and $c_{n}$ is the specific heat of each medium $n$. Consider further that this system is subjected to a point heat source placed at $\mathrm{O} \boldsymbol{x}_{s}=\left(x_{s}, y_{s}, z_{s}\right)$.

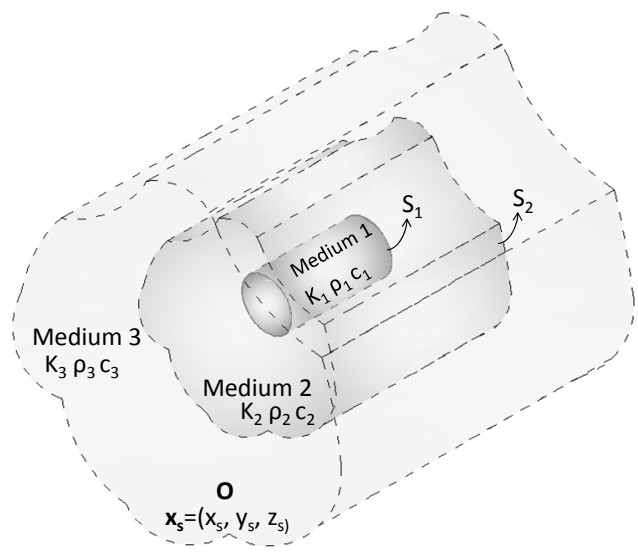

Figure 1: Three-dimensional geometry of the problem.

The incident temperature field produced at $\boldsymbol{x}=(x, y, z)$ by this heat source can be expressed by

$$
T_{\text {inc }}\left(\boldsymbol{x}, \boldsymbol{x}_{s}, \omega\right)=\frac{\mathrm{e}^{-\mathrm{i} \sqrt{-\frac{\mathrm{i} \omega}{K_{3}}} r_{0}}}{2 \lambda_{3} r_{0}}
$$

in which $\omega$ is the oscillating frequency, $i=\sqrt{-1}$ and $r_{0}=\sqrt{\left(x-x_{s}\right)^{2}+\left(y-y_{s}\right)^{2}+\left(z-z_{s}\right)^{2}}$. 


\section{Boundary element formulation (BEM)}

The transient heat transfer by conduction to calculate the heat $\left(T^{(n)}\right)$ at any point of the spatial 3D homogeneous solid domain is governed by the diffusion equation:

$$
\left(\frac{\partial^{2}}{\partial x^{2}}+\frac{\partial^{2}}{\partial y^{2}}+\frac{\partial^{2}}{\partial z^{2}}\right) T^{(n)}(\boldsymbol{x}, \omega)+\left(k_{c n}\right)^{2} T^{(n)}(\boldsymbol{x}, \omega)=0
$$

in which $k_{c n}=\sqrt{\frac{-\mathrm{i} \omega}{K_{n}}}$.

The boundary integral equation, formulated in the frequency domain, can be constructed by applying the reciprocity theorem, leading to the following boundary equations:

a) along the domain of the implanted body (Medium 1)

$$
\begin{array}{r}
b T^{(1)}\left(\boldsymbol{x}_{0}, \omega\right)=\int_{S_{1}} q^{(1)}\left(\boldsymbol{x}, \mathbf{n}_{n 1}, \omega\right) G^{(1)}\left(\boldsymbol{x}, \boldsymbol{x}_{0}, \omega\right) \mathrm{d} s \\
-\int_{S_{1}} H^{(1)}\left(\boldsymbol{x}, \mathbf{n}_{n 1}, \boldsymbol{x}_{0}, \omega\right) T^{(1)}(\boldsymbol{x}, \omega) \mathrm{d} s
\end{array}
$$

b) along the domain of the inclusion (Medium 2)

$$
\begin{aligned}
b T^{(2)}\left(\boldsymbol{x}_{0}, \omega\right) & =\int_{S_{1}} q^{(2)}\left(\boldsymbol{x},-\mathbf{n}_{n 1}, \omega\right) G^{(2)}\left(\boldsymbol{x}, \boldsymbol{x}_{0}, \omega\right) \mathrm{d} s \\
& +\int_{S_{2}} q^{(2)}\left(\boldsymbol{x}, \mathbf{n}_{n 2}, \omega\right) G^{(2)}\left(\boldsymbol{x}, \boldsymbol{x}_{0}, \omega\right) \mathrm{d} s \\
& -\int_{S_{1}} H^{(2)}\left(\boldsymbol{x},-\mathbf{n}_{n 1}, \boldsymbol{x}_{0}, \omega\right) T^{(2)}(\boldsymbol{x}, \omega) \mathrm{d} s \\
& -\int_{S_{1}+S_{2}} H^{(2)}\left(\boldsymbol{x}, \mathbf{n}_{n 2}, \boldsymbol{x}_{0}, \omega\right) T^{(2)}(\boldsymbol{x}, \omega) \mathrm{d} s
\end{aligned}
$$

c) along the exterior domain (Medium 3)

$$
\begin{aligned}
b T^{(3)}\left(\boldsymbol{x}_{0}, \omega\right) & =\int_{S_{2}} q^{(3)}\left(\boldsymbol{x},-\mathbf{n}_{n 2}, \omega\right) G^{(3)}\left(\boldsymbol{x}, \boldsymbol{x}_{0}, \omega\right) \mathrm{d} s \\
& -\int_{S_{2}} H^{(3)}\left(\boldsymbol{x},-\mathbf{n}_{n 2}, \boldsymbol{x}_{0}, \omega\right) T^{(3)}(\boldsymbol{x}, \omega) \mathrm{d} s+T_{\mathrm{inc}}\left(\boldsymbol{x}_{0}, \boldsymbol{x}_{s}, \omega\right)
\end{aligned}
$$


where $G^{(n)}$ and $H^{(n)}$ are respectively the fundamental solutions (Green's functions) for the temperature $\left(T^{(n)}\right)$ and heat flux $\left(q^{(n)}\right)$, at a point $\boldsymbol{x}=(x, y, z)$ on the boundary $S_{1}$ and $S_{2}$, due to a virtual point heat source at $\boldsymbol{x}_{0}=\left(x_{0}, y_{0}, z_{0}\right) ; \mathbf{n}_{n 1}$ and $\mathbf{n}_{n 2}$ represent the unit outward normal along the boundary $S_{1}$ and $S_{2}$, respectively, at $\boldsymbol{x}=(x, y, z) ; b$ is a constant defined by the shape of the boundary, taking the value $1 / 2$ if $\boldsymbol{x}_{0}=\left(x_{0}, y_{0}, z_{0}\right) \in S$, and 1 otherwise.

The required Green's functions for temperature and heat flux in an unbounded medium, in Cartesian coordinates, are given by:

$$
\begin{gathered}
G^{(n)}\left(\boldsymbol{x}, \boldsymbol{x}_{0}, \omega\right)=\frac{\mathrm{e}^{-\mathrm{i} k_{c n} r}}{4 \lambda_{n} \pi r} \\
H^{(n)}\left(\boldsymbol{x}, \mathbf{n}_{n 1}, \boldsymbol{x}_{0}, \omega\right)=\frac{\mathrm{e}^{-i k_{c r} r}\left(-\mathrm{i} k_{c n} r-1\right)}{4 \lambda_{n} \pi r^{2}} \frac{\partial r}{\partial \mathbf{n}_{n 1}} \\
H^{(n)}\left(\boldsymbol{x}, \mathbf{n}_{n 2}, \boldsymbol{x}_{0}, \omega\right)=\frac{\mathrm{e}^{-\mathrm{i} k_{c n} r}\left(-\mathrm{i} k_{c n} r-1\right)}{4 \lambda_{n} \pi r^{2}} \frac{\partial r}{\partial \mathbf{n}_{n 2}},
\end{gathered}
$$

with $r=\sqrt{\left(x-x_{0}\right)^{2}+\left(y-y_{0}\right)^{2}+\left(z-z_{0}\right)^{2}}$

The global solution is found by solving eqns (3), (4) and (5), which requires the discretization of the interfaces $S_{1}$ and $S_{2}$ into $N$ planar boundary elements, with one nodal point in the middle of each element.

The integrations in eqn (6) are evaluated using a Gaussian quadrature scheme when the element to be integrated is not the loaded element. For the loaded element (the singular element), however, the integrands exhibit a singularity and the integration can be carried out in closed form, as will be demonstrated.

Consider the singular rectangular element of width $\mathrm{W}$ (in the $x$ direction) and length $\mathrm{L}$ (in the $z$ direction) shown in Figure 2.

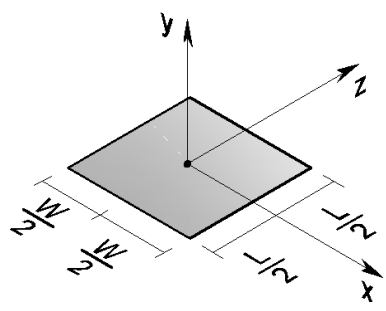

Figure 2: Scheme of a planar boundary element. 
Since in this case $r$ is perpendicular to the normal (e.g. $\boldsymbol{r} \mathbf{n}_{n 1}=0, \boldsymbol{r} \mathbf{n}_{n 2}=0$ ), the singular term $\quad \int_{-L / 2}^{L / 2} \int_{-W / 2}^{W / 2} H^{(n)}\left(\boldsymbol{x}, \mathbf{n}_{n 1}, \boldsymbol{x}_{0}, \omega\right) d x d z \quad$ and $\int_{-L / 2}^{L / 2} \int_{-W / 2}^{W / 2} H^{(n)}\left(\boldsymbol{x}, \mathbf{n}_{n 2}, \boldsymbol{x}_{0}, \omega\right) d x d z$ disappears. On the other hand, the integration of the Green's function $\int_{-L / 2}^{L / 2} \int_{-W / 2}^{W / 2} G^{(n)}\left(\boldsymbol{x}, \boldsymbol{x}_{0}, \omega\right) d x d z$ leads to a singular term.

This integration is evaluated by first expressing $G^{(n)}\left(\boldsymbol{x}, \boldsymbol{x}_{0}, \omega\right)$ as the sum of two-dimensional Green's functions with varying spatial wavenumbers.

This procedure allows the integration of $\int_{-L / 2}^{L / 2} \int_{-W / 2}^{W / 2} G^{(m)}\left(\boldsymbol{x}, \boldsymbol{x}_{0}, \omega\right) d x d z$ to be obtained as

$$
\int_{-L / 2}^{L / 2} \int_{-W / 2}^{W / 2} G^{(n)}\left(\boldsymbol{x}, \boldsymbol{x}_{0}, \omega\right) d x d z=\frac{-\mathrm{i}}{4 \lambda_{n} L_{v s}} \sum_{m=-M}^{M} \int_{-L / 2}^{L / 2} I_{1}\left(k_{c n z} \bar{r}_{0}\right) \mathrm{e}^{-\mathrm{i} k_{z m} z} d z
$$

with

$$
\int_{-L / 2}^{L / 2} I_{1}\left(k_{c n z} \bar{r}_{0}\right) \mathrm{e}^{-\mathrm{i} k_{z m} z} d z=I_{1}\left(k_{c n z} \bar{r}_{0}\right) L \quad \text { if } \quad m=0
$$

or $\quad \int_{-L / 2}^{L / 2} I_{1}\left(k_{c n z} \bar{r}_{0}\right) \mathrm{e}^{-\mathrm{i} k_{z m} z} d z=I_{1}\left(k_{c n z} \bar{r}_{0}\right) \frac{2 \sin \left(k_{z m} \frac{L}{2}\right)}{k_{z m}}$ if $\quad m \neq 0$

where $I_{1}\left(k_{c n z} \bar{r}_{0}\right)=\int_{-W / 2}^{W / 2} H_{0}\left(k_{c n z} \bar{r}_{0}\right) d x$

$I_{1}\left(k_{c n z} \bar{r}_{0}\right)$ is calculated analytically, following the expressions in Tadeu et al. $[20,21]$,

$$
\begin{aligned}
\int_{0}^{W / 2} H_{0}\left(k_{c n z} \bar{r}_{0}\right) d x & =\frac{W}{2} H_{0}\left(k_{c n z} \frac{W}{2}\right)+ \\
\pi & \frac{L}{4}\left[H_{1}\left(k_{c n z} \frac{W}{2}\right) S_{0}\left(k_{c n z} \frac{W}{2}\right)-H_{0}\left(k_{c n z} \frac{W}{2}\right) S_{1}\left(k_{c n z} \frac{W}{2}\right)\right]
\end{aligned}
$$

where $S_{n s}(\ldots)$ are Struve functions of order $n s$.

\section{Verification of the BEM formulation}

In this section, the proposed algorithm is verified using two circular cylindrical concentric inclusions, embedded in an unbounded space, aligned along the $z$ axis (see Figure 3), for which analytical solutions can be derived. A point heat source placed at $\left(x_{s}, y_{s}, z_{s}\right)$ is assumed to excite the medium. Continuity of heat 
temperatures and heat fluxes are prescribed on the interfaces between inclusions and on the exterior boundary. To enable comparison with the 3D BEM model, the length of the inclusion is limited by imposing null heat fluxes on sections $z=0.0 \mathrm{~m}$ and $z=L_{t}$.

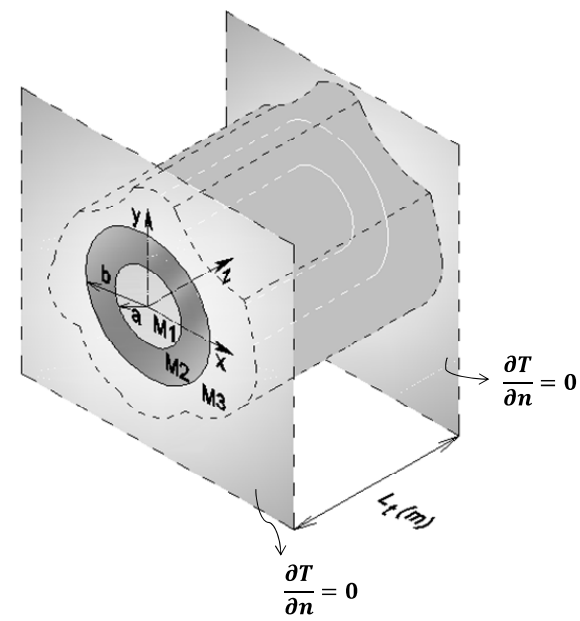

Figure 3: Two circular cylindrical concentric inclusions embedded in an unbounded space, with length limited by adiabatic sections.

The analytical solution for this problem is obtained by first applying a spatial Fourier transformation in the $z$ direction, which allows the solution to be obtained as the sum of two-dimensional solutions with a varying spatial wavenumber in that direction. The null normal heat fluxes at sections $z=0.0 \mathrm{~m}$ and $z=L_{t}$ are accomplished by adding the temperature field generated by the real source to that produced by virtual sources (image sources), which are placed in the $z$ direction and act as mirrors of the real source, so as to ensure the required boundary conditions.

$$
T^{(n)}\left(\boldsymbol{x}, \boldsymbol{x}_{s}, \omega\right)=\frac{2 \pi}{L_{v s}} \sum_{m=-\infty}^{\infty} \hat{T}^{(n)^{\prime}}\left(x, y, x_{s}, y_{s}, k_{z m}, \omega\right)
$$

with $L_{v s}$ is the spatial source interval, and $k_{z m}=\frac{2 \pi}{L_{v s}} m(m=1, \ldots, N S)$ and $\hat{T}^{(n)^{\prime}}\left(x, y, x_{s}, y_{s}, k_{z m}, \omega\right)=\hat{T}^{(n)}\left(x, y, x_{s}, y_{s}, k_{z m}, \omega\right)\left[\mathrm{e}^{-i k_{z m} z_{m 0}}+\sum_{m=1}^{N S_{z}} \sum_{j=1}^{4} \mathrm{e}^{-\mathrm{i} k_{z m} z_{m j}}\right]$ where $\quad z_{m 0}=z-z_{s}, \quad z_{m 1}=z+z_{0}-2 L_{t} m, \quad z_{m 2}=z+z_{0}+2 L_{t}(m-1)$, $z_{m 3}=z-z_{0}-2 L_{t} m$ and $z_{m 4}=z-z_{0}+2 L_{t} m$. 
The number of virtual sources $N S_{z}$ to be used in the calculations is defined so that the signal responses can be correctly computed in the time frame, which is determined by the frequency increment $1 / \Delta f$. This procedure does not introduce any type of error into the computed time impulse response within the time window defined. Notice that $L_{v s}$ should be at least twice the distance from the real source to the farthest virtual source.

The accuracy of the BEM solution has been studied by computing the response at two receivers located in the system as illustrated in Figure 4 $[\operatorname{rec} 1(-0.05,0.0,1.0) \mathrm{m}, \operatorname{rec} 2(-0.4,0.0,0.9) \mathrm{m}]$.

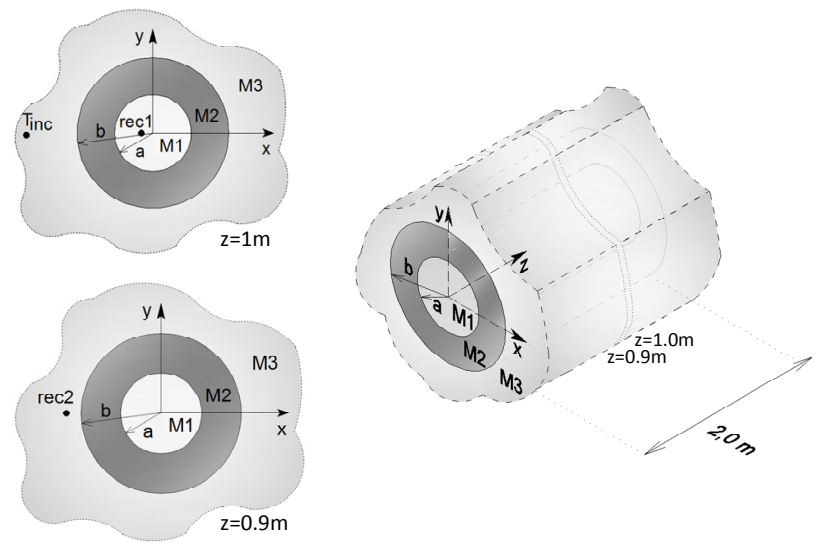

Figure 4: Scheme of the two receivers placed at two concentric cylindrical inclusions embedded in an unbounded space [rec1 $(-0.05,0.0,1.0) \mathrm{m}, \operatorname{rec} 2(-0.4,0.0,0.9) \mathrm{m}]$, subjected to a $3 \mathrm{D}$ point heat source, $T_{i n c}$.

The material thermal properties of the cylindrical inclusion (Medium 2) with radius of $b=0.30 \mathrm{~m}$ composed by an inner body (Medium 1) with radius of $a=0.15 \mathrm{~m}$ and of the hosting uniform solid medium (Medium 3) are listed in Table 1. $L_{v s}$ is assumed to be $60.0 \mathrm{~m}$. The system is $2 \mathrm{~m}$ in length and limited by adiabatic surfaces.

Table 1: $\quad$ Thermal material properties.

\begin{tabular}{|c|c|c|c|}
\hline & $\begin{array}{c}\text { Thermal conductivity, } \lambda \\
\left(\mathrm{W} \cdot \mathrm{m}^{-1} \cdot{ }^{\mathrm{o}} \mathrm{C}^{-1}\right)\end{array}$ & $\begin{array}{c}\text { Density, } \rho \\
\left(\mathrm{kg} \cdot \mathrm{m}^{-3}\right)\end{array}$ & $\begin{array}{c}\text { Specific heat, } c \\
\left(\mathrm{~J} \mathrm{~kg}^{-1} \cdot{ }^{\mathrm{o}} \mathrm{C}^{-1}\right)\end{array}$ \\
\hline Medium 1 & 0.12 & 712.0 & 1550.0 \\
\hline Medium 2 & 63.9 & 7832.0 & 434.0 \\
\hline Medium 3 & 1.4 & 2300.0 & 880.0 \\
\hline
\end{tabular}


The system was subjected to a three dimensional point heat source, $T_{\text {inc }}$, placed at $(-1.0 \mathrm{~m}, 0.0 \mathrm{~m}, 1.0 \mathrm{~m})$. Temperature computations were performed in the frequency range $\left[0.0,1 \times 10^{-5}\right] \mathrm{Hz}$. The responses were computed for complex frequencies $\omega_{c}=\omega-\mathrm{i} \eta \quad$ (with $\eta=0.7 \Delta \omega, \Delta \omega=2 \times \pi \times \Delta f \quad$ and $\left.\Delta f=0.5 \times 10^{-7} \mathrm{~Hz}\right)$.

The responses have been computed both analytically and using the 3D BEM formulation, considering a different number of boundary elements.

Figures $5 \mathrm{a}$ and $5 \mathrm{c}$ show the analytical response (real and imaginary parts) at the two receivers. Figures $5 \mathrm{~b}$ and $5 \mathrm{~d}$ illustrate the absolute value of the error at receiver 1 and 2 when the problem is solved using the proposed BEM algorithm, using two different numbers of boundary elements to discretize the inclusions: 800 and 1800. It can be observed that the solution improves as the number of boundary elements increases. As expected, the BEM error is higher at the receiver 2 , since it is placed nearest to the heat source.

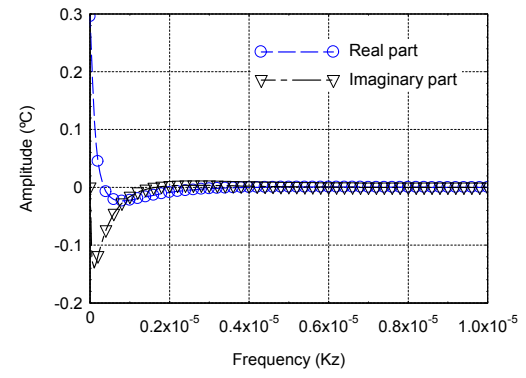

a)

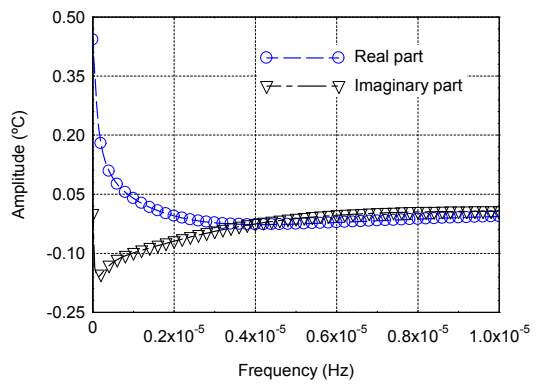

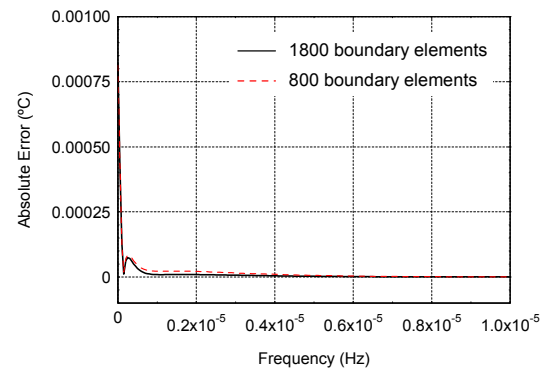

b)

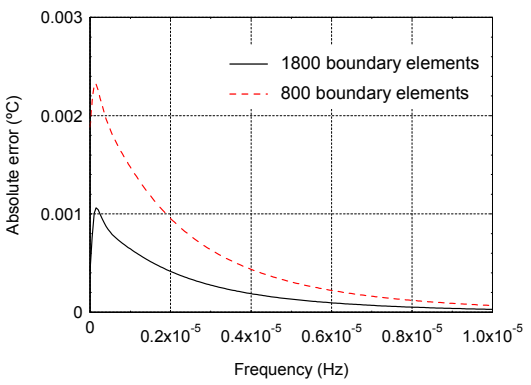

d)

Figure 5: Analytical solutions and BEM error results at two receivers: rec1 analytical results; b) rec1 - variation of the BEM error with the frequency; c) rec2 - analytical results; d) rec2 - variation of the BEM error with the frequency.

For the same problem described before, the temperature responses were computed at one grid of receivers placed at $z=1.0 \mathrm{~m}$, crossing the system. 
Figure 6 illustrates the responses obtained. This figure shows the real and imaginary parts of the analytical response and the error obtained when the system is solved using the BEM. It can be seen that the magnitude of the error increases as the receivers approach the inclusion's boundary. On the other hand, as expected, the BEM accuracy improves as the receivers are placed further away from the heat source. It can further be observed that the solution improves as the number of boundary elements increases, which illustrates the good accuracy of the BEM response.
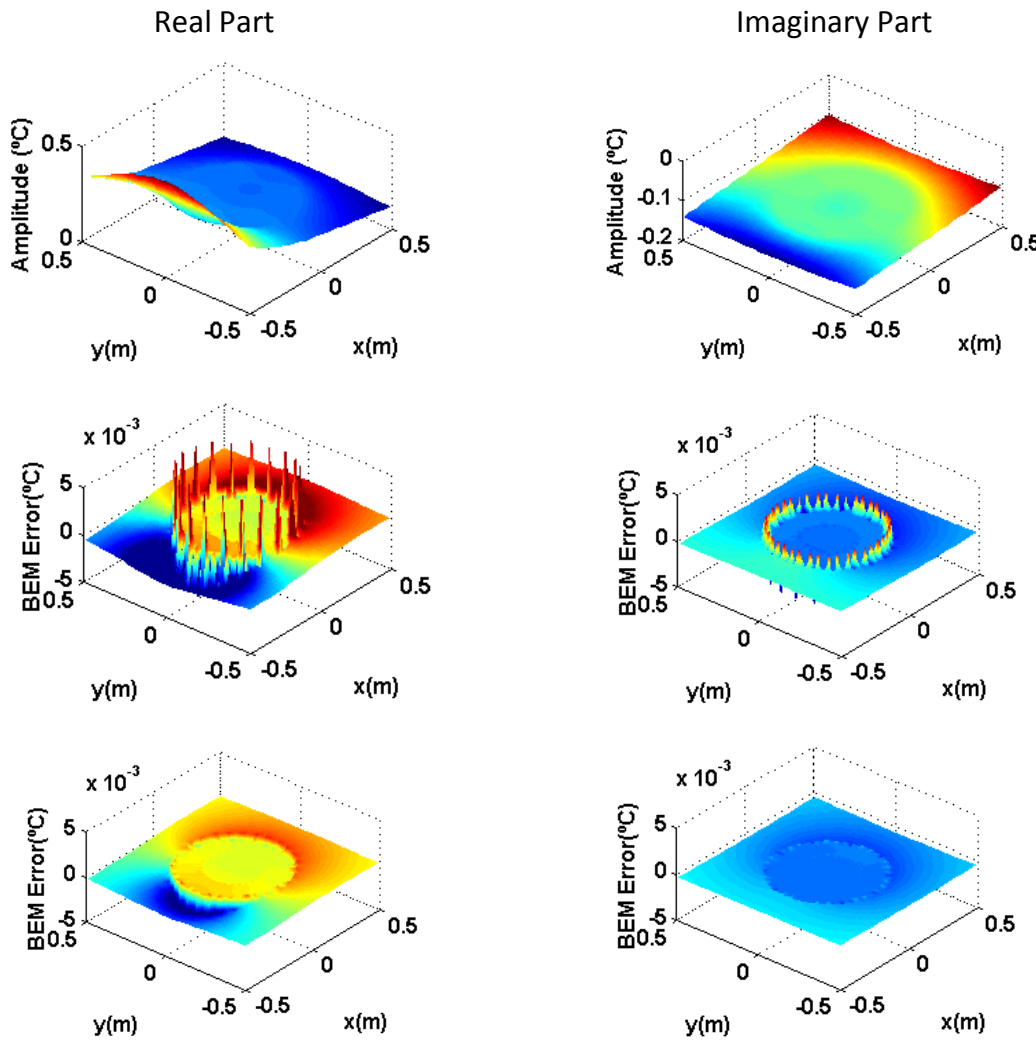

a)

b)

Figure 6: Comparative analysis of analytical and BEM responses (real and imaginary parts), considering different numbers of boundary elements: a) analytical response; b) error using BEM (with 800 boundary elements); c) error using BEM (with 1800 boundary elements).

\section{Conclusions}

In this paper the authors presented the simulation of the three-dimensional heat transfer by conduction using a 3D BEM formulation. Analytical integrations 
were given when the element to be integrated is the loaded one (singular element).

Then, the 3D BEM solution, incorporating the analytical solutions of the singular integrals, have been verified against analytical solutions derived for two concentric cylindrical circular inclusions limited by two perpendicular sections, where null heat fluxes where imposed: the responses showed very good accuracy.

\section{Acknowledgements}

The research work presented herein was supported by FEDER funds through the Operational Programme for Competitiveness Factors - COMPETE and by national funds through the FCT - Portuguese Foundation for Science and Technology), under research project PTDC/ECM/114189/2009. The presented work is also framed under the Energy for Sustainability Initiative of the University of Coimbra, through the MIT PORTUGAL, supported by the FCT.

\section{References}

[1] Carslaw, HS. and Jaeger, J.C., Conduction of Heat in Solids. Oxford University Press, Vivian Ridler, Walton Street, Oxford, second edition, 1959.

[2] Bathe, K.J., Numerical Methods in Finite Element Analysis. Prentice-Hall, New Jersey, 1976.

[3] Ozisik, M.N., Finite Difference Methods in Heat Transfer. CRC Press Inc, USA, 1994.

[4] Juncu, Gh., Unsteady conjugate forced convection heat/mass transfer from a finite flat plate. International Journal of Thermal Sciences, 47: 972-984, 2008 .

[5] Brebbia, C.A., Telles, J.C. and Wrobel, L.C., Boundary Elements Techniques: Theory and Applications in Engineering. Springer-Verlag, Berlin-New York, 1984.

[6] Ochiai, Y., Steady heat conduction analysis in orthotropic bodies by triplereciprocity BEM. Computer Modeling in Engineering and Sciences, 2(4): 435-446, 2001.

[7] Šarler, B., Towards a mesh-free computation of transport phenomena. Engineering Analysis with Boundary Elements, 26(9): 731-738, 2002.

[8] Ma, F., Chatterjee, J., Henry, DP. and Banerjee, P.K., Transient heat conduction analysis of 3D solids with fiber inclusions using the boundary element method. International Journal for Numerical Methods in Engineering, 73: 1113-1136, 2008.

[9] Jablonski, P., Integral and geometrical means in the analytical evaluation of the BEM integrals for a 3D Laplace equation. Engineering Analysis with Boundary Elements, 34: 264-273, 2010.

[10] Xianyun, Qin., Zhang, J., Xie, G., Zhou, F. and Li, G., A general algorithm for the numerical evaluation of nearly singular integrals on $3 \mathrm{D}$ boundary 
element. Journal of Computational and Applied Mathematics, 235(14): 4174-4186, 2011.

[11] Zhou, H., Niu, Z., Cheng, C. and Guan, Z., Analytical integral algorithm applied to boundary layer effect and thin body effect in BEM for anisotropic potential problems. Computers \& Structures, 86: 1656-1671, 2008.

[12] Telles, J.C.F., A self-adaptive co-ordinates transformation for efficient numerical evaluation of general boundary element integrals. International Journal for Numerical Methods in Engineering, 24: 959-973, 1987.

[13] Chen, X.L. and Liu, Y.J., An advanced 3-D boundary element method for characterizations of composite materials. Engineering Analysis with Boundary Elements, 29: 513-523, 2005.

[14] Hayami, K., Variable transformations for nearly singular integrals in the boundary element method. Publications of the Research Institute for Mathematical Sciences, 41: 821-842, 2005.

[15] Ma, H. and Kamiya, N., Distance transformation for the numerical evaluation of near singular boundary integrals with various kernels in boundary element method. Engineering Analysis with Boundary Elements, 26: 329-339, 2002.

[16] Ma, H. and Kamiya, N., A general algorithm for the numerical evaluation of nearly singular boundary integrals of various orders for two- and threedimensional elasticity. Computational Mechanics, 29: 277-88, 2002.

[17] Ma, H. and Kamiya, N., Distance transformation for the numerical evaluation of near singular boundary integrals with various kernels in boundary element method. Engineering Analysis with Boundary Elements, 26: 329-339, 2002.

[18] Srivastava, D.N., Contractor, Efficient evaluation of integrals in 3-D Boundary Element Method using linear shape functions over plane triangular elements. Applied Mathematical Modelling, 16(6): 282-290, 1992.

[19] Niu, Z., Wendland, W.L., Wang, X.X. and Zhou, H.L., A semi-analytical algorithm for the evaluation of the nearly singular integrals in threedimensional boundary element methods. Computer Methods in Applied Mechanics and Engineering, 194: 1057-74, 2005.

[20] Tadeu, A., Santos, PFA. and Kausel, E., Closed-form integration of singular terms for constant, linear and quadratic boundary elements. Part I. SH wave propagation. Engineering Analysis with Boundary Elements, 23: 671-681, 1999.

[21] Tadeu, A., António, J. and Castro, I., Coupling the BEM/TBEM and the MFS for the numerical simulation of acoustic wave propagation. Engineering Analysis with Boundary Elements, 34: 405-416, 2010. 